\title{
AUTOESTIMA Y ANSIEDAD EN ESTUDIANTES UNIVERSITARIOS SEXUALMENTE ACTIVOS
}

\author{
Bárbara Sofía Córdova Witeczek ${ }^{1}$ \\ Universidad Rafael Urdaneta - Escuela de Psicología -Venezuela \\ Edgar Andrés Córdova Witeczek² \\ Universidad del Zulia - Facultad de Medicina - Escuela de Medicina- \\ Venezuela
}

\section{Resumen}

En el contexto de esta investigación, la ansiedad y la autoestima se perfilan como unas de las variables más importantes a comprobar en personas activas sexualmente, en tal sentido, se ha centrado el interés de la indagación en estudiantes universitarios sexualmente activos con edades que van desde los 19 a 30 años. El objetivo general fue determinar la relación entre autoestima y ansiedad en esta población de estudiantes ubicada en el municipio Maracaibo del Estado Zulia - Venezuela, para ello fue necesario identificar el nivel de autoestima y describir el nivel de ansiedad en la muestra seleccionada, procediendo luego a establecer la relación entre las variables. El tipo de investigación fue transaccional, correlacional, y su diseño de corte no experimental. Para la variable autoestima se utilizó la escala de autoestima (AESTI), la cual es una escala tipo Likert, de 10 ítems, con 6 puntos; elaborada por Rosenberg (1965a), para la variable ansiedad se utilizó el Inventario de Ansiedad de Beck -1976- (Beck, Epstein y Cols, 1988), conformado por 21 ítems, cada uno compuesto por cuatro modalidades de respuesta que van de cero (0), no ansiedad, a tres (3) intensidad severa de ansiedad; se aplicó el instrumento adaptado al medio venezolano por el Centro de Investigaciones Psicológicas de la Facultad de Medicina de la Universidad de los Andes en Venezuela. Se concluye que existe una fuerte relación entre las variables, por

1 Psicóloga, Universidad Rafael Urdaneta Estado Zulia, Maracaibo, Venezuela, terapeuta en ejercicio, Correo: barbaracordova106@hotmail.com.

2 Médico Cirujano. Facultad de Medicina. Universidad del Zulia, Maracaibo- Venezuela. Correo: ecordovawiteczek@gmail.com. 
cuanto la autoestima, al integrar los aspectos cognitivos, afectivos y conductuales, se ve afectada por factores psicológicos, personales, propios de la personalidad del individuo; además de factores sociales y familiares, los cuales pudieran incidir en la percepción del individuo en cuanto a sus competencias y su valoración personal, entre otros aspectos.

Palabras clave: ansiedad en jóvenes, sexualidad, autoestima, salud mental, estudiantes universitarios.

\section{Abstract}

Anxiety and self-esteem would be outlined as one of the most important variables to check in sexually active people, in this context are of interest in this research university students sexually active from 19 to 30 years. This research defined its general objective; To determine the relationship between self-esteem and anxiety in this population of students in the Maracaibo municipality of Zulia State, Venezuela, for this was necessary; Identify the level of self-esteem and describe the level of anxiety in university students. The type of research was non-experimental correlational transactional. For the variable of selfesteem was used the scale of self-esteem (AESTI) which is a scale type Likert of 10 items with 6 points elaborated by Rosenberg (1965a), for the anxiety variable was used Beck's anxiety Inventory (1976-), For 21 items, each composed of four response modalities ranging from zero (0), not anxiety, to three (3) severe anxiety intensity, the instrument adapted to the Venezuelan medium was applied by the Center for Psychological Research of the Faculty of Medicine of The University of the Andes in Venezuela. It is concluded that there is a strong relationship between the variables, because the self-esteem, integrating the cognitive, affective and behavioral aspects, is affected by psychological factors, personal personality of the individual, as well as factors Social and family, which could affect the perception of the individual in terms of their competencies and personal assessment, among other aspects.

Keywords: youth anxiety, self-esteem, sexuality and self-esteem, mental health, university students. 


\section{Introducción}

A lo largo de las investigaciones en psicología, los puntos de atención han sido los trastornos psicológicos y cómo éstos afectan las distintas esferas del individuo. Por tanto, son relativamente recientes los estudios encaminados a la comprobación de los factores que favorecen las áreas social y emocional, siendo la variable autoestima una de las más estudiadas, debido a los efectos de esta en el bienestar del ser humano, hasta tal punto que se ha denominado una necesidad humana básica. Es Rosenberg (1965a) quien la cataloga como una necesidad, pues el autor señala como razón para ello que una alta autoestima se asocia a personas sanas y felices, mientras que la baja autoestima se asocia a personas insatisfechas y desconcertadas.

La autoestima es definida como la apreciación que una persona tiene de sí misma, la cual puede ser positiva o negativa, pues está basada en un eje afectivo y otro cognitivo, dado que el sujeto se siente de alguna manera determinado por lo que piensa acerca de sí mismo. Respecto a las variables que parecen influir en los niveles de autoestima destacan, de manera general: la apariencia y las capacidades con relación al cuerpo, los logros académicos y/o laborales, el clima familiar y la medida en que las necesidades sociales alcanzan un alto de grado de satisfacción (Craighead, McHale y Pope, 2001). Con relación a esta última, se señalan aspectos como la amistad, las habilidades de interacción con los pares y el establecimiento de las relaciones de pareja productivas, importantes y dignas; es decir, es uno de los factores que abarca tanto el área emocional como social del individuo y, es precisamente este, el que se asocia con la importancia de la filiación y la decisión de activar el comportamiento sexual para cubrir tanto las necesidades biológicas como las sociales.

Ser sexualmente activo, es denominado por la Organización Mundial de la Salud (OMS, 2010) como parte de la salud sexual o, bien, el estado de bienestar físico, mental y social en relación con la sexualidad, el cual requiere un enfoque positivo y respetuoso de la sexualidad y de las relaciones sexuales, del mismo modo, la posibilidad de tener experiencias sexuales placenteras y seguras, libres de toda coacción, discriminación y violencia.

Según la misma OMS (2006) los jóvenes entre 15 y 24 años solamente representan el $25 \%$ de la población con vida sexual activa. En 
el caso de Venezuela, aunque no existen datos específicos, registrados por los organismos oficiales, sobre la incidencia de la población que es sexualmente activa se conoce que la mayoría de los jóvenes inician su sexualidad de manera precoz (Corona, 2014). Ahora bien, desde la perspectiva psicológica, social y cultural, el preámbulo de una vida sexual activa se inicia con información insuficiente respecto a la sexualidad, tabúes sobre esta, resistencia y poco conocimiento con respecto al uso de los métodos anticonceptivos y sentimientos de omnipotencia característicos en la mayoría de los adolescentes.

No obstante, lo anterior también parece extrapolarse a la adultez temprana. En un estudio realizado con jóvenes universitarios se encontró que a pesar de la formación académica y de la creciente disponibilidad de información acerca de sexualidad, el tabú imperante acerca todo lo que rodea las relaciones entre los sexos, provoca que los estudiantes posean información insuficiente o poco específica acerca del funcionamiento de su cuerpo y de las consecuencias en la salud, lo que los conduce a una práctica sexual inadecuada (Chávez, Petrzelová y Zapata, 2009).

Adicional a esto, McKinney (citado por Allen, 2000) argumenta que la decisión de mantener una vida sexualmente activa puede deberse a diversos factores tanto positivos como negativos: el placer físico que se obtiene, expresión de amor, necesidad de filiación, para probar la autonomía e independencia, por presión social, entre otros.

En este sentido, vivenciar relaciones sexuales en el marco de la juventud, pudiera depender del nivel de autoestima de estos; así, las razones positivas para mantenerlas parecen asociarse a una alta estima, mientras que las razones negativas se asociarían a una baja estima (Allen, 2000). Todo ello, considerando los planteamientos de Rosenberg (1965a), quien sostiene que las personas que actúan sobre la base de una autoestima insuficiente se muestran limitadas en sus relaciones, de igual modo, sumisas a la voluntad de otros; todo ello, dada la inseguridad que experimentan en sus interacciones, dando muestra de problemas en el autoconcepto y el autocontrol, áreas en las que buscan la aprobación de otros, lo cual puede llevarlos a realizar conductas no probas3.

3 Para ampliar consúltese Bandura, A. y Cervone, D., (1983). Self-evaluative and self-efficacy mechanisms governing the motivational effects of goal systems, Journal of Personality and Social Psychology 45, pp. 1017-1028. 
Dicho de otro modo, cuando el joven decide ser sexualmente activo por motivos como el placer físico que se obtiene, asumiendo éste último de manera responsable, la necesidad de expresarle amor a la pareja a través de ello y/o la necesidad de filiación, es sinónimo de que se muestra seguro en su vida afectiva, manifestando habilidades de comunicación y respeto hacía sí mismo y hacia su pareja, lo cual conforma criterios claves de una alta autoestima (Agudelo, Casadiegos, Sánchez, 2009).

A la par, la sexualidad sana y responsable se asocia también con el aumento de la autoestima (Leal y Prato, 2007; Ceballos, Barliza y León, 2008), lo cual es una clara prueba de las influencias bidireccionales de la autoestima y la sexualidad activa. Sin embargo, en el comportamiento sexual también intervienen otros factores que, de hecho, están vinculados con la información que se maneje de esta, ello pudiera ser motivo de ansiedad (Rodríguez, 2010). La ansiedad, en este contexto, según las argumentaciones de Beck y Clark (1988), se referiría a un intenso malestar interior debido a la actividad sexual y el sentimiento que tiene el sujeto de no ser capaz de controlar los sucesos futuros, como consecuencia de esta; estos criterios estarían acompañados por síntomas físicos como tensión muscular, molestias estomacales, entre otros.

Para la OMS (2006), los jóvenes constituyen el grupo más vulnerable en relación con las consecuencias negativas de mantener relaciones sexuales. Se ha comprobado que el comportamiento sexual atenúa la ansiedad, es decir, que ser sexualmente activos produce un efecto de tipo ansiolítico o atenuante del estrés, que se manifiesta tanto en la conducta de los sujetos, como a niveles fisiológicos o neuroendocrinos (Justel, Bentosela y Mustaca, 2009); no obstante, pudiera provocar los efectos contrarios cuando se mantienen de manera desinformada e impulsados por una baja autoestima. De acuerdo con Leary y Dobbins (citado por Rodríguez, 2010) los sujetos ansiosos presentan una tendencia a subestimar sus capacidades para tener interacciones sexuales, son propensos a autocriticarse cuando interactúan con el otro sexo, anticipan como negativas las consecuencias de una situación y se autoreprochan con mayor frecuencia por no haber actuado correctamente en una interacción con alguien del otro sexo.

Efectivamente, la autoestima y la ansiedad se perfilarían como unas de las variables más importantes a comprobar en personas activas sexualmente. Parece que ambas se influencian entre sí, y sus niveles estarían 
estrechamente vinculados con el grado de satisfacción de las prácticas sexuales. Por ello, los jóvenes universitarios corresponderían al grupo de mayor interés, tomando en consideración que es aquel con mayor actividad sexual, y a su vez, con mayor riesgo.

De los planteamientos precedentes surgió la necesidad de responder la siguiente interrogante: ¿Cómo es la autoestima y ansiedad en estudiantes universitarios heterosexuales activos sexualmente? Partiendo de las reflexiones iniciales la pregunta formulada se desagregó en otras interrogantes específicas, esto conllevó a determinar la relación entre autoestima y ansiedad en estudiantes universitarios heterosexuales activos sexualmente. En concordancia con las interrogantes, los objetivos que guiaron la búsqueda se plantearon en los siguientes términos: Determinar la relación entre autoestima y ansiedad en esta población de estudiantes ubicada en el municipio Maracaibo del Estado Zulia- Venezuela, lo cual implicó identificar el nivel de autoestima en estudiantes heterosexuales activos sexualmente; describir el nivel de ansiedad en estudiantes universitarios activos sexualmente, para luego establecer la relación entre las citadas variables

En relación al sistema educativo venezolano, particularmente en el subsector educación superior, la sexualidad no es un tema estudiado ampliamente, aún menos lo concerniente a variables psicológicas como son las aquí indagadas. Al estudiar la relación entre la autoestima y la ansiedad en personas heterosexuales activas sexualmente se abren las posibilidades de ampliar el conocimiento sobre este tema, del mismo modo, profundizar la importancia de la adecuada educación sexual en el país. Por otra parte, se pretendió estimar, la posible influencia de estas variables psicológicas, autoestima y ansiedad, considerando la sexualidad en el marco del desarrollo de una alta autoestima o de niveles de ansiedad, como aspectos importantes para la salud desde una visión integral. 


\section{Metodología}

La investigación llevada a cabo fue de tipo descriptivo, de nivel perceptivo, ya que se buscó medir las variables ansiedad y autoestima. Según Hernández, Fernández y Baptista (1998), este tipo de investigación procura especificar las propiedades importantes del fenómeno objeto de estudio. Se recurrió al trabajo de campo para la aplicación de los instrumentos diseñados, en el presente caso la aplicación se realizó en el espacio de la Universidad Rafael Urdaneta ubicada en Maracaibo- Estado Zulia- Venezuela ${ }^{4}$.

\section{Diseño de la investigación}

El diseño de la investigación es correlacional, ya que su propósito fue medir el grado de relación de dos o más conceptos o variables, las cuales, en esta investigación son autoestima y ansiedad en un contexto particular. $\mathrm{X} 1 \rightarrow \mathrm{X} 2, \mathrm{X} 1$.. Variable 1 (Autoestima) - X2: Variable 2 (Ansiedad).

\section{Población}

La población es definida como el conjunto de todos los casos que concuerdan con una serie de especificaciones (Selltiz, citado por Hernández et al., 2006). La investigación se llevó a cabo en el periodo transcurrido de mayo (2016) a octubre (2017), con estudiantes universitarios heterosexuales activos sexualmente en edades comprendidas entre 19 y 30 años, localizados en Maracaibo, Estado Zulia- Venezuela. La variable autoestima se investigó tomando en cuenta los postulados de Rosenberg (1965a) y la ansiedad considerando la teoría de Beck (Beck et al., 1988).

\section{Muestra}

En la investigación se empleó un muestreo de tipo no probabilístico, que según Parra (2003), corresponde a procedimientos de selección de

4 Consideraciones Éticas: En la presente investigación se tomó en cuenta y estuvo sustentada por el Código de Ética Profesional del Psicólogo de Venezuela (1996), específicamente por los siguientes artículos: Artículo 54: Este artículo plantea que la investigación en la Psicología debe inspirarse y basarse en los más elevados principios éticos y científicos La investigación estuvo basada en los principios éticos y científicos de la investigación Artículo 55: Refiere que la investigación deberá ser realizada y supervisada por personas cualificadas y entrenadas en el ámbito científico. 
muestras en donde intervienen factores distintos al azar. De igual manera, dentro de este tipo de muestreo, se selecciona el muestreo accidental en cual el investigador selecciona los sujetos para componer la muestra, de acuerdo a la accesibilidad. (Tamayo y Tamayo, 1994). Para efectos de esta investigación la muestra estuvo conformada por 120 estudiantes universitarios heterosexuales activos sexualmente de ambos sexos, cursantes de diferentes carreras universitarias.

\section{Definición operacional de las variables}

La autoestima: se define como el puntaje arrojado por el análisis de la Escala de Autoestima de Rosenberg $(1962)^{5}$, mide con escalas, donde estos arrojan resultados de autoestima elevada, autoestima media y por último autoestima baja, basándose en tres dimensiones: cognoscitiva, afectiva y conductual. La Ansiedad: Consiste en el puntaje medio obtenido en el Inventario de Ansiedad de Beck (Beck, \& Emery, 1985), que mide con escalas donde estos indican un nivel normal, nivel moderado, nivel de moderado a severo y por último un nivel severo de ansiedad, basándose en cuatro áreas: cognitiva, emocional, conductual y fisiológica.

\section{Técnicas de recolección de datos y descripción de los instrumentos}

Para la variable de autoestima se aplicó la escala de autoestima AESTI la cual es una escala tipo Likert de 10 ítems, con 6 puntos, elaborada por Rosenberg (1965a). Los ítems 1, 2, 3, 4 y 5 están destinados a identificar la autoestima alta, y los ítems 6, 7, 8, 9 y 10 están destinados a identificar la autoestima baja; proponiendo como alternativas de respuesta "completamente de acuerdo", "moderadamente de acuerdo", "ligeramente de acuerdo", "ligeramente en desacuerdo", "moderadamente en desacuerdo" y "completamente en desacuerdo". Esta se centra en la medición de sentimientos de respeto y aceptación hacia sí mismo, dando como resultados autoestima baja, autoestima media y autoestima alta, en la investigación se aplicó el instrumento adaptado al medio venezolano por el Centro de Investigaciones Psicológicas de la Facultad de Medicina

5 Para ampliar véase también Coopersmith, S. (1990). The antecedents of Self-esteem. Consulting Psycologists Press 3ra. Edition. E.E.U.U. 
de la Universidad de los Andes (ULA), en Venezuela, según la cual las puntuaciones van desde autoestima alta a autoestima baja.

Tabla 1

Forma de Corrección AESTI

\begin{tabular}{ll}
\hline Completamente en desacuerdo & Ligeramente de acuerdo. \\
Moderadamente en desacuerdo & Moderadamente de acuerdo. \\
Ligeramente en desacuerdo & Completamente de acuerdo. \\
\hline
\end{tabular}

Fuente: Rosenberg (1965a)

Tabla 2

Baremos de interpretación AESTI

\begin{tabular}{ll}
\hline Autoestima & Rango - Percentil \\
Autoestima Alta & Mayor o igual a 41 \\
Autoestima Media & De 25 a 40 \\
Autoestima Baja & Menor o igual a 25 \\
\hline
\end{tabular}

Fuente: Rosenberg (1965a)

\section{Inventario de ansiedad de Beck (1976)}

Para la variable de ansiedad se utilizó el Inventario de Ansiedad de Beck -1976- (Beck y Clark, 1988), el cual está conformado por 21 ítems, cada uno compuesto por cuatro modalidades de respuesta que van de cero (0), no ansiedad, a tres (3) intensidad severa de ansiedad, indicando lo siguiente:

- Cero (0) no existe sintomatología;

- Uno (1) intensidad leve del síntoma;

- $\operatorname{Dos}(2)$, intensidad moderada del síntoma;

- $\operatorname{Tres}(3)$ : Intensidad severa del síntoma. ${ }^{6}$

6 Se miden aspectos fisiológicos que denotan y expresan la ansiedad en el sujeto como palpitaciones, sudoración y sensaciones de malestar, de igual manera evalúa aspectos emocionales como miedo y temor relacionados a episodios que provoquen más ansiedad. Del total de 21 ítems, 13 están destinados a medir los síntomas físicos y fisiológicos (ítems n: $1,2,3,6,7,11,12,13,15,18,19,20,21$ ), 5 a medir los aspectos cognitivos (ítems $n^{\circ}: 4$, $5,10,14,16)$, y 3 tienen una connotación tanto física como cognitiva (ítems n: 8, 9 y 17). 
La aplicación fue individual, los sujetos valoraron cada reactivo considerando el grado en que se sentían molestos, por el síntoma particular, colocaron una X en la columna que representa el grado de ansiedad que este poseía. La corrección del inventario se realizó así: en la primera columna, las respuestas tienen un valor de 0 puntos; en la segunda columna tienen un valor de 1 punto; en la tercera columna tienen un valor de 2 puntos en la cuarta y última columna, las respuestas tienen un valor de 3 puntos; posteriormente estos valores se suman y se interpretan.

Tabla 3

Forma de corrección del BAI

\begin{tabular}{ll}
\hline Cero (0) & No existe sintomatología \\
Uno (1) & Intensidad leve del síntoma \\
Dos (2) & Intensidad moderada del síntoma \\
Tres (3) & Intensidad severa del síntoma \\
\hline
\end{tabular}

Fuente: Beck y Emery (1985).

Tabla 4

Baremos de interpretación del BAI

\begin{tabular}{lcccc}
\hline Nivel de Ansiedad & $\begin{array}{c}\text { Puntaje } \\
\text { Global }\end{array}$ & Fisiológico & Cognitivo & Emocional \\
\hline Normal & $0-9$ & $0-4$ & $0-2$ & $0-1$ \\
Leve a Moderada & $10-18$ & $5-9$ & 3.5 & $2-3$ \\
Moderada a Severa & $19-29$ & $10-18$ & $6-8$ & $4-5$ \\
Severa & $30-63$ & $19-39$ & $9-15$ & Mayor a 5 \\
\hline
\end{tabular}

Fuente: Beck y Emery (1985).

\section{(1965) \\ Propiedades psicométricas: Escala AESTI por Rosenberg}

Al momento de medir la escala AESTI, Silver y Teppett (1965) encontraron que la escala correlacionada entre .56 y .83 medidas clínicas similares con una muestra de 44 sujetos, puntuaciones que representar una validez adecuada. En 1965, la escala AESTI fue puesta a prueba en 5024 estudiantes dando como resultado mediante el coeficiente de reproductividad Guttman una puntuación de .92, la cual demuestra que esta es adecuada. En cuanto al Alpha de Crombach arrojó 0.799 lo que indica, de igual de manera, un buen índice de confiabilidad. 


\section{Inventario de ansiedad por Beck (1976)}

Con respecto a la validez Beck et al. (1988) reportaron buena consistencia interna, dando 0.92 de Alfa de Crombach, siendo esta adecuada y consistente para la medición de la variable. En relación a la confiabilidad, ha sido obtenida por diferentes métodos. En el caso de confiabilidad TestRetest, Spilberg (1971), demostró que la misma se ubica entre 0.92 - 0.94. Por otro lado, Beck y Emery (1985) planteó la correlación de partición por mitades ubicándose estas en 0.78 y 0.92, lo cual refleja alta consistencia de respuesta.

\section{Discusión}

Desde esa óptica, el presente estudio tiene como finalidad determinar la relación entre autoestima y ansiedad en estudiantes universitarios activos sexualmente, problema que resulta importante para la salud y muy concretamente para la salud mental y sus implicaciones en el estado de salud general, para conocer las motivaciones y aspectos psico-afectivos que condicionan el comportamiento de estos importantes actores (sujetos de 19 a 30 años, estudiantes sexualmente activos y de orientación sexual heterosexuales), en un entorno complejo que exige cada día la disposición de asumir con madurez la sexualidad y, el compromiso con su desarrollo y crecimiento personal. Al respecto, el procesamiento de los datos permitió a la investigadora generar un conjunto de tablas que sistematizan los resultados en relación a las variables, dimensiones e indicadores.

A continuación, se presenta la Tabla 5, dentro de la cual se describen los valores máximos, promedio y mínimos, que arrojó la sumatoria de los datos para los individuos que conformaron la muestra de estudio, los cuales interpretados a través de baremo de Rosenberg (1965a), permitieron identificar el nivel de autoestima promedio general. 
Tabla 5

Resultados generales variable autoestima

\begin{tabular}{lcc}
\hline \multicolumn{1}{c}{ Descripción } & Resultado & Categoría \\
\hline Sumatoria máxima & 45 & Autoestima alta \\
Sumatorio promedio & 35,47 & Autoestima media \\
Sumatoria mínima & 23 & Autoestima baja \\
\hline
\end{tabular}

Fuente: elaboración propia.

Como se observa en la Tabla 5, la suma promedio alcanzada por los encuestados se encuentra de acuerdo al baremo de Rosenberg (1965a), en un nivel medio, al ubicarse la mayoría de los estudiantes, en un valor de 35.47. Esto indica una tendencia en cuanto a la opinión de estos, con relación al manejo de los aspectos cognitivos, afectivos y conductuales, los datos permiten reforzar la opinión de sí mismos, de igual modo, alcanzar un nivel de satisfacción con sus logros. Obsérvese, en este sentido, coincidencia en cuanto al manejo de los ítems, estos permiten reforzar su autopercepción, pues, tanto los reactivos positivos, como los negativos, los ubican en niveles intermedios, situación que refuerza su apreciación en cuanto a la presencia de una autoestima moderada.

Al igual que la actitud, comprender la autoestima exige de la integración de tres aspectos fundamentales, a saber: lo que la persona piensa acerca de sus potenciales y logros, es decir, la percepción de sí mismo; en segundo lugar, lo que expresa a través de sus emociones y sentimientos en relación a estos importantes aspectos y, en tercer lugar, las conductas convertidas en acciones concretas para materializar esos resultados, a manera de lograr condición de armonía, equilibrio, auto reconocimiento y autocontrol.

De acuerdo con Rosenberg (1993), la autoestima es un conjunto de percepciones, pensamientos, evaluaciones, sentimientos y tendencias de comportamiento dirigidas hacia nosotros mismos, hacia nuestra manera de ser y hacia los rasgos de nuestro cuerpo y nuestro carácter. En resumen: es la percepción evaluativa de nosotros mismos, citando a Rogers (1967), máximo exponente de la psicología humanista, la raíz de los problemas de muchas personas es que se desprecian y se consideran seres sin valor e indignos de ser amados; de ahí la importancia que le concedía a la aceptación incondicional del cliente. En efecto, el concepto de autoestima se aborda desde entonces en la escuela humanista como un derecho inalienable de 
toda persona, sintetizado en el siguiente «axioma»: Todo ser humano, sin excepción, por el mero hecho de serlo, es digno del respeto incondicional de los demás y de sí mismo; merece estimarse a sí mismo y que se le estime.

En el marco de esas ideas, se presentan en la Tabla 6, los resultados promedio y de desviación alcanzados para la variable autoestima. En ese sentido, se presentan los hallazgos arrojados por el procesamiento de los cuestionarios .con respecto al objetivo específico número uno, orientado a identificar el nivel de autoestima en estudiantes universitarios activos sexualmente.

Tabla 6

Variable: Autoestima

\begin{tabular}{lcc}
\hline \multirow{2}{*}{ Dimensiones } & Estudiantes & \\
\cline { 2 - 3 } & Promedio & Desviación \\
\hline Unidimensional & 3,55 & 0,84 \\
Promedio General & 3,55 & 0,84 \\
\hline
\end{tabular}

Fuente: elaboración propia.

La variable autoestima obtuvo un promedio en las respuestas de los estudiantes de 3,55, con una baja dispersión de 0,84, representando de acuerdo a los baremos de interpretación una moderada autoestima. Al respecto, Rosenberg (1993) plantea que la autoestima se basa en una apreciación que puede ser positiva o negativa de sí mismo, apoyada en una base afectiva o cognitiva, ya que el individuo se siente de una manera determinada, a partir de lo que este piensa de sí mismo; es decir, una baja autoestima refleja un rechazo, una insatisfacción y un menosprecio de sí mismo.

Sobre la base de lo planteado y mediante la interpretación de los datos obtenidos sobre la variable, se puede señalar que la autoestima es el reflejo que la persona tiene de sí misma y el sentimiento del individuo de sentirse valioso, así como digno de respeto. Además, los autores consideran a la autoestima como un estado de bienestar y una fuerza que le permite al individuo valorarse y responsabilizarse por su vida, aspecto importante que, de acuerdo a los resultados, no ha sido alcanzado de manera satisfactoria por los estudiantes, pues la investigación revela un moderado desarrollo de los aspectos cognitivos, afectivos y conductuales necesarios para reforzar 
la opinión de sí mismos, la seguridad y el autorrespeto como elementos determinantes de una adecuada autoestima.

Continuando con el análisis e interpretación de los resultados, se le proporcionó respuesta al segundo objetivo específico, formulado para describir el nivel de ansiedad en estudiantes universitarios activos sexualmente; los hallazgos se presentan en las tablas subsiguientes, a partir de la valoración de la citada variable y sus dimensiones.

Tabla 7

Variable: Ansiedad

\begin{tabular}{lcc}
\hline \multicolumn{1}{c}{ Dimensiones } & \multicolumn{2}{c}{ Estudiantes } \\
\cline { 2 - 3 } & Promedio & Desviación \\
\hline Área Cognitiva & 0,41 & 0,71 \\
Área Emocional & 0,66 & 0,77 \\
Área Conductual & 2,49 & 0,51 \\
Área Fisiológica & 0,33 & 0,63 \\
Promedio General & 0,97 & 0,66 \\
\hline
\end{tabular}

Fuente: elaboración propia.

En lo que respecta a la variable ansiedad, se obtuvo un promedio para los estudiantes de 0,97, con una baja dispersión en las respuestas $(0,66)$, manifestando un nivel leve de ansiedad al plantear que no poseen ningún tipo de temor y pocas veces se sienten nerviosos: solo cuando hay situaciones demasiado estresantes en las que aparecen levemente los nervios y otros síntomas.

Según Beck y Clark (1988), la ansiedad se refiere a un estado emocional desagradable de carácter subjetivo, connotado por sensaciones de desagrado como tensión o nerviosismo, acompañado de igual manera por síntomas fisiológicos como temblor, fatiga, mareo, entre otros. Por otra parte, Kaplan y Sadock (1996), plantean que la ansiedad se refiere a un estado emocional desagradable en el que existen sensaciones de peligro amenazador, caracterizado por malestar, tensión o aprehensión. Este estado está acompañado por una descarga del Sistema Nervioso Autónomo, implicando síntomas físicos incontrolables, tales como aumento del ritmo respiratorio, palidez, aumento del sudor, sensación de debilidad, entre otros. 
Para Freud (1926), la palabra ansiedad se introdujo en el lenguaje como una traslación del término freudiano "agnst", que significaba angustia, siendo esta una combinación de afecto negativo y arousal fisiológico. Dicho esto, planteaba que la ansiedad neurótica era una manifestación de los conflictos inconscientes de cada individuo.

Tabla 8

Dimensión: Área Cognitiva

\begin{tabular}{lcc}
\hline \multirow{2}{*}{ Indicadores } & \multicolumn{2}{c}{ Estudiantes } \\
\cline { 2 - 3 } & Promedio & Desviación \\
\hline Temor a que suceda lo peor & 0,65 & 0,90 \\
Temor a perder el control & 0,34 & 0,71 \\
Temor a morir & 0,23 & 0,53 \\
Promedio General & 0,41 & 0,71 \\
\hline
\end{tabular}

Fuente: elaboración propia.

Para la dimensión área cognitiva, se obtuvo un promedio en las respuestas de los encuestados de 0,41 , con una muy baja dispersión $(0,71)$, reflejando, según los baremos construidos en el marco metodológico, que los mismos no presentan un nivel leve o bajo de ansiedad, debido a que, mediante sus planteamientos, no poseen temor ni descontrol en sus actividades académicas.

Beck y Clark (1988), plantean que los patrones de pensamiento contraproducente y distorsionado, así como conductas desadaptativas, son aquellas que generan un estado ansioso y disturbios emocionales. Las personas que sufren de ansiedad tienden a sobreestimar el grado de peligro y la probabilidad de daño en una situación específica, así como a minimizar sus habilidades para manejar las situaciones que puedan exponerse como una amenaza a su bienestar físico o psicológico. Profundizando en el análisis de los resultados se puede observar -en lo que respecta a los indicadores temor a que suceda lo peor, temor a perder el control y temor a morir, que existen promedios en las respuestas de los encuestados de 0,65, 0,34 y 0,23, respectivamente, con bajas dispersiones de 0,90,0,71 y 0,53, reflejando igualmente para todos la inexistencia de algún tipo de temor por parte de los estudiantes en sus actividades, de acuerdo a las respuestas expresadas en el instrumento y procesadas a través de estadística descriptiva. 
Según McInnis (1998), la transición de la escuela a la universidad puede resultar estresante, el autor argumenta que durante el primer año las perspectivas, valores y patrones de comportamiento son determinados con respecto a la educación superior. La salud mental de estudiantes universitarios ha sido foco de estudios mediante investigaciones en las que se han concluido que los estudiantes están sujetos a incrementar los estresores, poniendo así en riesgo su bienestar psicológico.

Tabla 5

Dimensión: área emocional

\begin{tabular}{lcc}
\hline \multirow{2}{*}{ Indicadores } & \multicolumn{2}{c}{ Estudiantes } \\
\cline { 2 - 3 } & Promedio & Desviación \\
\hline Aterrorizado & 0,48 & 0,71 \\
Nervioso & 0,84 & 0,80 \\
Asustado & 0,64 & 0,80 \\
Promedio General & 0,66 & 0,77 \\
\hline
\end{tabular}

Fuente: elaboración propia.

Con respecto a la dimensión área emocional, se obtuvo un promedio para los encuestados de 0,66, con una baja dispersión en las respuestas de 0,77 , reflejando los mismos que no presentan afecciones en el área emocional, negando sentirse asustados o nerviosos en su quehacer diario. Asimismo, y soportado en los planteamientos expuestos, se pueden observar los promedios para los indicadores Aterrorizado, Nervioso y Asustado, los cuales igualmente presentan un nivel bajo o leve, con promedios de 0,48, 0,84 y 0,64 , planteando que muy pocas veces se sienten nerviosos o con miedo en las instituciones.

Beck (1990), señala que las respuestas del organismo ante una amenaza se expresan en tres tipos de reacción: movilización, inhibición o desmovilización. En cuanto a la movilización, esta prepara al individuo para una defensa activa y puede ser observado en los diversos sistemas. En cuanto a la inhibición, es una expresión de parálisis y está diseñada para ganar tiempo y encontrar una estrategia apropiada con respecto a la situación, en cuanto al orden cognitivo, en esta etapa existe un bloqueo de funciones como el razonamiento, concentración, objetividad. En el área conductual, existe una paralización de movimientos espontáneos, especialmente de los músculos faciales. Finalmente, en cuanto a la desmovilización se refiere 
a una paralización del aparato motriz y reflejo, el sentido de desamparo ante el enfrentamiento de una amenaza abrumadora; normalmente, va acompañada de desmayo, disminución de la presión sanguínea y de los latidos del corazón.

\section{Tabla 6}

Dimensión: área conductual

\begin{tabular}{lcc}
\hline \multirow{2}{*}{ Indicadores } & \multicolumn{2}{c}{ Estudiantes } \\
\cline { 2 - 3 } & Promedio & Desviación \\
\hline Inquietud & 4,94 & 0,84 \\
Desmayo & 0,03 & 0,18 \\
Promedio General & 2,49 & 0,51 \\
\hline
\end{tabular}

Fuente: elaboración propia.

Con respecto a la dimensión área conductual, se obtuvo un promedio para los encuestados de 2,49, con una baja dispersión en las respuestas de 0,51, evidenciando un nivel moderado de ansiedad. Resulta necesario profundizar el análisis detallando los indicadores de la dimensión, los cuales son Inquietud, con un promedio de 4,94, reflejando un nivel severo de ansiedad producido por una elevada inquietud; y Desmayo con un promedio de 0,03, evidenciando casi la inexistencia de este en el quehacer de los estudiantes. Dichos datos se interpretaría a partir de lo indicado por Beck y Clark (1988), quienes plantean que los patrones de pensamiento contraproducente y distorsionado, así como conductas desadaptativas, son aquellas que generan un estado ansioso y disturbios emocionales. Las personas que sufren de ansiedad tienden a sobreestimar el grado de peligro y la probabilidad de daño en una situación específica, asimismo, a minimizar sus habilidades para manejar las situaciones que puedan exponerse como una amenaza a su bienestar físico o psicológico. 
Tabla 7

Dimensión: área fisiológica

\begin{tabular}{lcc}
\hline \multirow{2}{*}{ Indicadores } & \multicolumn{2}{c}{ Estudiantes } \\
\cline { 2 - 3 } & Promedio & Desviación \\
\hline Adormecimiento o cosquilleo & 0,03 & 0,18 \\
\hline Sentirse acalorado & 0,27 & 0,63 \\
\hline Piernas tambaleantes & 0,46 & 0,94 \\
\hline Incapacidad para relajarme & 0,24 & 0,58 \\
\hline Mareo & 1,00 & 1,00 \\
\hline Taquicardia & 0,34 & 0,69 \\
\hline Sensación de ahogo & 0,27 & 0,71 \\
\hline Mano temblorosa & 0,20 & 0,53 \\
\hline Escalofríos & 0,26 & 0,60 \\
\hline Temor a perder el control & 0,34 & 0,26 \\
\hline Indigestión o molestia & 0,50 & 0,91 \\
estomacal & 0,14 & 0,45 \\
\hline Rostro sonrojado & 0,29 & 0,70 \\
\hline Sudoración & 0,33 & 0,63 \\
\hline Promedio General &
\end{tabular}

Fuente: elaboración propia.

En lo referente a la dimensión área fisiológica, se obtuvo un promedio para los encuestados de 0,33, con una baja dispersión en las respuestas de 0,63, reflejando que no existe ningún tipo de ansiedad que altere el área fisiológica, descartando la presencia de esos síntomas para el estudiantado en la labor universitaria.

Para soportar lo expuesto por los estudiantes en la interpretación de los instrumentos fue necesario reflejar que, aunque parezca sorprendente, la ansiedad en niveles normales es saludable, pues este es un mecanismo de supervivencia básico, que nos alerta de amenazas y peligros para prepararnos para la lucha o la huida. Para Barlow (2001), la ansiedad empieza a ser patológica cuando no desaparece; es decir, que pasada la situación amenazante esta no cesa; aun cuando sepamos que no hay nada en realidad por qué sentirnos temerosos, continuamos sintiendo la ansiedad. 
En lo que refiere a los indicadores de la dimensión, se puede observar que casi todos presentan un promedio bajo, a excepción del indicador mareo, que presenta un promedio de 1,00, con un nivel leve, lo que refleja la inexistencia de síntomas como adormecimiento o cosquilleo, piernas tambaleantes, sensación de ahogo, escalofríos, rostro sonrojado, entre otros; y que en muy pocas ocasiones sienten mareos a causa de la ansiedad en sus actividades dentro de la institución. Asimismo, con respecto a las dispersiones en las respuestas, es necesario acotar que la mayoría refleja un promedio bajo, exceptuando algunos indicadores, que muestran ciertas diferencias no muy significativas en las opiniones emitidas por los encuestados.

Para la American Psicological Asociation (APA, 1994) la ansiedad es una emoción caracterizada por sentimientos de tensión, preocupación y cambios físicos como el aumento del flujo sanguíneo, así como pensamientos intrusivos. De acuerdo con las distintas definiciones anteriores, la ansiedad implica un estado emocional que se refleja en comportamientos como la inquietud, nerviosismo y temor, subyacente a un problema cognitivo (Beck y Clark, 1988) ello sirve de apoyo para la investigación, que está enfocada a medir la ansiedad mediante la teoría cognitiva-conductual.

Seguidamente, corresponde dar respuesta al último objetivo específico orientado a establecer la relación entre la autoestima y la ansiedad presente en estudiantes universitarios sexualmente activos; en base a los cálculos obtenidos al aplicar el programa estadístico Spss ${ }^{7}$, continuación en la Tabla 8, se muestran los resultados de correlación Rho de Spearman, procedimiento seleccionado para establecer el nivel de relación entre las variables:

\section{Tabla 8}

Resultados de la correlación entre las variables (Rho de Spearman)

\begin{tabular}{lc}
\hline Variable & Resultado \\
\hline Ansiedad & 0.61 \\
Autoestima & \\
\hline
\end{tabular}

Fuente: elaboración propia.

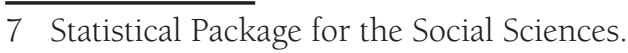


Como se observa en la tabla, el cálculo de correlación, a través del software estadístico Spss, arrojó un valor de -0.61, ubicándose en una categoría negativa fuerte, lo cual indica relación inversamente proporcional entre las variables, razón por la cual valores de ansiedad generan condiciones que pudieran afectar la percepción de los estudiantes en cuanto a su autopercepción y valoración, pues constituyen factores generadores de estrés. Esto revela que al aumentar los niveles de ansiedad pudiera afectarse la autoestima de los estudiantes participantes en el estudio, lo cual, de acuerdo a Rosenberg (1993) está relacionado con su capacidad para enfrentar los cambios y problemas inherentes a su rol o ejercicio.

La condición de fuerte es una expresión de la importante relación que existe entre las variables, lo cual no es determinante; es decir, que la ansiedad no es el único factor que pudiera incidir en la autoestima de los estudiantes. Este resultado, se sustenta en las aportaciones de Arciniegas y Bigott (2004) al expresar que la autoestima, al integrar los aspectos cognitivos, afectivos y conductuales, se ve afectada por factores psicológicos, personales propios de la personalidad del individuo; además de factores sociales y familiares, que pudieran incidir en la percepción del individuo en cuanto a sus competencias, valoración personal, entre otros aspectos.

\section{Conclusiones}

La vida del ser humano resulta casi siempre un desafío para los estudiosos de la conducta y sus diversas acepciones. Su capacidad natural de adaptarse a los cambios y la necesidad de sentirse bien, lo conducen muchas veces a través del desbloqueo de sus represiones hacia los instintos sexuales, generar claridad en la utilización de sus energías con total libertad, logrando alcanzar un feliz desempeño. Sin embargo, no siempre resulta tan fácil, y es por ello que las diversas investigaciones se enfocan hacia el estudio de los trastornos que, desde el punto de vista psicológico, afectan los procesos en los que el individuo se desenvuelve.

En ese sentido, la autoestima pasa a ser una necesidad básica del individuo, debido a los efectos que puede generar en el mismo, regulando su comportamiento. Por otra parte, la ansiedad representa para muchos autores, una combinación entre miedo y preocupación, que además de 
forma frecuente, viene acompañada de diversas sensaciones físicas, y es generada por la baja autoestima, influyendo en cómo las personas toman sus decisiones, su estabilidad emocional, su energía, su habilidad para aprender de los errores, su capacidad para desenvolverse sexualmente, entre otros.

Con relación a lo anterior, la presente investigación tuvo como objetivo general determinar la relación entre autoestima y ansiedad en estudiantes universitarios activos sexualmente, del Municipio Maracaibo, Estado Zulia. Partiendo del análisis de los resultados alcanzados, luego de la aplicación de los instrumentos de recolección de datos a la muestra de estudio, se llegó a las siguientes conclusiones:

Con relación a la variable autoestima, la suma promedio alcanzada por los encuestados, se encontró de acuerdo al baremo de Rosenberg (1965b), en un nivel medio; lo cual indica una tendencia moderada de los encuestados, con relación al manejo de los aspectos cognitivos, afectivos y conductuales. Esto permite reforzar la opinión de sí mismos y alcanzar un nivel de satisfacción con sus logros.

Para profundizar, se recurrió al análisis de los resultados alcanzados para cada objetivo específico. En este sentido, para el objetivo orientado a identificar el nivel de autoestima en estudiantes universitarios activos sexualmente, del Municipio Maracaibo del Estado Zulia; se concluyó que existe una apreciación moderada de los estudiantes hacia sí mismos, en cuanto a sentirse valiosos y dignos de respeto. Este resultado, representa un reflejo moderado de lo que los estudiantes consideran sea su estado de bienestar y la fuerza que les permite valorarse a sí mismos y responsabilizarse por su vida.

Con respecto al objetivo específico orientado a describir el nivel de ansiedad en estudiantes universitarios activos sexualmente, el análisis de los resultados alcanzados permitió concluir para la variable ansiedad, que existe en un nivel leve. Significando que los estudiantes no poseen altos temores, y pocas veces se sienten nerviosos. Solo cuando hay situaciones estresantes en las áreas académica, conductual, emocional y fisiológica, aparecen levemente los nervios y otros síntomas.

Finalmente, en relación con el último objetivo específico, orientado a establecer la relación entre la autoestima y la ansiedad, presente en estudiantes 
universitarios sexualmente activos; el análisis de los resultados alcanzados permitió concluir que existe una relación inversamente proporcional y fuerte entre las variables ansiedad y autoestima. Es proporcional, por cuanto valores de ansiedad generan condiciones que pudieran afectar la percepción de los estudiantes con respecto a su autopercepción y valoración, pues constituyen factores generadores de estrés. Esto indica que al aumentar los niveles de ansiedad, pudiera afectarse la autoestima de los estudiantes integrantes de la muestra, lo cual, según la opinión de Rosenberg (1993), está relacionado con su capacidad para enfrentar los cambios y los problemas inherentes a su rol o ejercicio.

En atención a los hallazgos, se pudo determinar que existe una fuerte relación entre las variables, pues la autoestima, al integrar los aspectos cognitivos, afectivos y conductuales, se ve afectada por factores psicológicos, personales propios de la personalidad del individuo, además de factores sociales y familiares, los cuales pudieran incidir en la percepción del individuo en cuanto a sus competencias y su valoración personal, entre otros aspectos.

\section{Referencias}

Agudelo Vélez, D., Casadiegos, C., Sánchez, D. (2009). Características de ansiedad y depresión en estudiantes universitarios International Journal of Psychological Research, 1(1). Universidad de San Buenaventura Medellín, Colombia.

Allen, R. (2000). Factores que influyen en la decisión de inicio de la vida sexual en adolescentes. (Tesis de Maestría). Nuevo León: Universidad Autónoma de Nuevo León. Recuperado de http://eprints.uanl. $\mathrm{mx} / 6462 / 1 / 1080111919$.PDF

APA. (1994). Diagnostic and statistical manual of mental disorders (4th. ed.). Washington D.C.

Arciniegas, M y Bigott, M. (2004). Niveles de autoestima, inteligencia emocional y estilo explicativo en niños con disfunción cerebral motora. Trabajo de grado. Universidad Rafael Urdaneta. Maracaibo.

Bandura, A. y Cervone, D. (1983). Self-evaluative and self-efficacy mechanisms governing the motivational effects of goal systems , Journal of Personality and Social Psychology 45, pp. 1017-1028. 
Barlow, D. (2001). Psicología Anormal. "Psicología anormal. Un enfoque integral" Barlow, D.H. \& Durand, Estados Unidos.

Beck, A, Emery, G. (1985). Anxiety disorders and phobias. Estados Unidos. Harper Collins Publisher.

Beck, Epstein, Brown G., \& Steer R. (1988). An Inventory for Measuring Clinical Anxiety: Psychometric Properties. J Consult Clin Psychol.

Beck, A. y Clark, D. (1988). Anxiety and depression: An information processing perspective. Anxiety research, 1, 23-36.

Beck. (1990). Terapia Cognitiva de la depresión. España. Editorial Desclee de Brouwer, S.A.

Ceballos, Barliza y León. (2008). Autoestima y disfunción sexual en estudiantes universitarios de programas de salud de Santa Marta. Colombia. Recuperado de: http://www.scielo.org.co/pdf/psdc/n22/ n22a05.pdf.

Chávez, M., Petrzelova, J. y Zapata, J. (2009). Actitudes respecto a la sexualidad en estudiantes universitarios. Enseñanza e Investigación en Psicología, 14(1): 161-175. Recuperado de http://www.redalyc. org/pdf/292/29214110.pdf. Recuperado de consulta 14 de febrero de 2018.

Coopersmith, S. (1990). The antecedents of Self-esteem. Consulting Psycologists Press 3ra Edition. E.E.U.U.

Corona, J. (2014). Embarazo a edad temprana y educación sexual en el contexto socioeducativo venezolano. Investigación arbitrada: 181-184. Recuperado de http://www.saber.ula.ve/bitstream/123456789/40748/3/art14.pdf.

Craighead, E. McHale, S. y Poper, A. (2001). Self-Esteen Enhancement With Desarrollo de la Autoestima y la Conciencia Moral en las Contradicciones de la Sociedad Contemporánea. Madrid: Universidad de Comillas.

Freud, S. (1926). Inhibitions, symptoms, and axiety. London. Hogarth Press.

Hernández, Fernandez y Baptista. (2006). Metodología de la Investigación. Editorial Mc Graw Hill. México.

Justel, N. Bentosela, M. y Mustaca, A. (2009). Comportamiento sexual y ansiedad. Revista Latinoamericana de Psicología, 41(3): 429-444. 
Recuperado de http://www.scielo.org.co/pdf/rlps/v4ln3/v4ln3a04. pdf.

Kaplan, H y Sadock, B. (1996). Tratado de Psiquiatría. Salvat Editores. Barcelona.

Leal y Prato. (2007). Autoestima y locus de control en adultos jóvenes activos y no activos sexualmente. (Tesis de pregrado), Universidad Rafael Urdaneta, Maracaibo, Venezuela.

McInnis C, James R. (1998). First year on campus: Diversity in the initial experiences of Australian undergraduates. Centre for the study of Higher Education. Universidad de Melbourne.

Organización Mundial de la Salud. (2006). Estadísticas sanitarias mundiales. Ginebra: Suiza.

Organización Mundial de la Salud. (2010). Uso de los derechos humanos en pro de la salud sexual y reproductiva: mejorar los marcos jurídicos y normativos. Ginebra: Suiza.

Parra, J. (2003). Guía de muestreo. Colección XLV aniversario FCES.

Rodríguez, O. (2010). Relación entre satisfacción sexual, ansiedad y prácticas sexuales. Pensamiento Psicológico, 7(14): 41-52. Recuperado de http://www.redalyc.org/pdf/801/80113673003.pdf.

Rogers, C. (1967). Psicoterapia y Relaciones Humanas. Ediciones Araguara. Madrid.

Rosenberg, M. (1965a). Escala de Autoestima, Instituto de Investigaciones Psicológicas de la Universidad de os Andes, Mérida.

Rosenberg, M. (1965b). Society and the adolescent self-image. Princeton, NJ: Princeton University Press.

Rosenberg, M. (1993). Desarrollo de la Autoestima. Editoriales Martínez Roca. Barcelona.

Silver y Tippett. (1965). Instituto de Investigaciones Psicológicas de la Universidad de los Andes, Mérida.

Spilberg, S. (1971). Inventario de Ansiedad. El manual moderno. México. Tamayo y Tamayo. (1994). El proceso de la Investigación científica. Editorial Limusa. 